\title{
Therapeutic potential of kakkalide for their anti-stroke and anti-hyperlipidemic effects: Biological importance of lactate dehydrogenase (LDH) and HMG-CoA reductase in medicine
}

\author{
Kanika PATEL, Dinesh Kumar PATEL*
}

Faculty of Health Sciences, Sam Higginbottom University of Agriculture, Technology and Sciences, Prayagraj, India

Introduction: Kakkalide have been isolated from the flower of Pueraria thunbergiana is metabolized into irisolidone by human intestinal microflora. Pueraria thunbergiana is a plant of Leguminosae family contain significant amount of kakkalide. Kakkalide have been well known for their bioactivities including hepatoprotective, anti-inflammatory and antihyperlipidemic activity.

Methods: Biological potential of kakkalide in the medicine for their therapeutic benefit against stroke of human being have been investigated through literature data analysis of various research work in the present investigation. Biological potential of kakkalide for their antihyperlipidemic effects on 3-hydroxy-3-methylglutaryl (HMG)-CoA reductase have been investigated through literature data analysis of various research works. Biological potential of kakkalide for the anti-stroke activity have been investigated through literature data analysis of current scientific research work.

Results: Literature data analysis of various scientific research works signified the biological potential of kakkalide in the medicine and other allied health sectors. Literature data analysis revealed the biological potential of kakkalide in the medicine for their antihyperlipidemic potential as it potently lowered the serum levels of total cholesterol and triglyceride in Trition WR1339-induced hyperlipidemic mice. Further in the literature significant effect has been also produced on the hyperlipidemic mice induced by high fat diet. Scientific data analysis of kakkalide revealed their significance potential in the medicine for their LDH inhibitory potential which signified their anti-stroke activity in the medicine.

Conclusions: Literature data analysis of different scientific research work signified the biological importance and therapeutic benefit of kakkalide for the treatment of cardiovascular disorders including stroke and hyperlipidemia. 